PROCEEDINGS OF THE

AMERICAN MATHEMATICAL SOCIETY

Volume 138, Number 10, October 2010, Pages 3521-3529

S 0002-9939(2010)10525-7

Article electronically published on June 3, 2010

\title{
A NEW APPROACH TO THE EXPANSION OF POSITIVITY SET OF NON-NEGATIVE SOLUTIONS TO CERTAIN SINGULAR PARABOLIC PARTIAL DIFFERENTIAL EQUATIONS
}

\author{
EMMANUELE DIBENEDETTO, UGO GIANAZZA, AND VINCENZO VESPRI
}

(Communicated by Matthew J. Gursky)

\begin{abstract}
Let $u$ be a non-negative solution to a singular parabolic equation of $p$-Laplacian type $(1<p<2)$ or porous-medium type $(0<m<1)$. If $u$ is bounded below on a ball $B_{\rho}$ by a positive number $M$, for times comparable to $\rho$ and $M$, then it is bounded below by $\sigma M$, for some $\sigma \in(0,1)$, on a larger ball, say $B_{2}$, for comparable times. This fact, stated quantitatively in this paper, is referred to as the "spreading of positivity" of solutions of such singular equations and is at the heart of any form of Harnack inequality. The proof of such a "spreading of positivity" effect, first given in 1992, is rather involved and not intuitive. Here we give a new proof, which is more direct, being based on geometrical ideas.
\end{abstract}

\section{INTRODUCTION AND MAIN RESULTS}

Let $E$ be an open set in $\mathbb{R}^{N}$ and for $T>0$ let $E_{T}$ denote the cylindrical domain $E \times(0, T]$. Consider quasi-linear, parabolic differential equations of the form

$$
\begin{aligned}
& u \in C_{\mathrm{loc}}\left(0, T ; L_{\mathrm{loc}}^{2}(E)\right) \cap L_{\mathrm{loc}}^{p}\left(0, T ; W_{\mathrm{loc}}^{1, p}(E)\right) \\
& u_{t}-\operatorname{div} \mathbf{A}(x, t, u, D u)=0 \quad \text { weakly in } E_{T}
\end{aligned} \quad \text { for } 1<p<2,
$$

where the function $\mathbf{A}: E_{T} \times \mathbb{R}^{N+1} \rightarrow \mathbb{R}^{N}$ is only assumed to be measurable and subject to the structure conditions

$$
\left\{\begin{array}{l}
\mathbf{A}(x, t, u, D u) \cdot D u \geq C_{o}|D u|^{p} \\
|\mathbf{A}(x, t, u, D u)| \leq C_{1}|D u|^{p-1}
\end{array} \quad \text { a.e. } \quad(x, t) \in E_{T},\right.
$$

where $C_{o}$ and $C_{1}$ are given positive constants. The homogeneous prototype of such a class of parabolic equations is

$$
u_{t}-\operatorname{div}|D u|^{p-2} D u=0, \quad 1<p<2, \quad \text { weakly in } E_{T} .
$$

These equations are termed singular since, for $1<p<2$, the modulus of ellipticity $|D u|^{p-2} \rightarrow \infty$ as $|D u| \rightarrow 0$.

The parameters $\left\{N, p, C_{o}, C_{1}\right\}$ are the data, and we say that a generic constant $\gamma=\gamma\left(N, p, C_{o}, C_{1}\right)$ depends upon the data if it can be quantitatively determined a priori only in terms of the indicated parameters. For $\rho>0$ let $B_{\rho}$ be the ball

Received by the editors October 19, 2009.

2010 Mathematics Subject Classification. Primary 35K65, 35K67, 35B65; Secondary 35 B45.

The first author was supported in part by NSF grant \#DMS-0652385.

(C)2010 American Mathematical Society 
of center the origin on $\mathbb{R}^{N}$ and radius $\rho$, and for $y \in \mathbb{R}^{N}$ let $B_{\rho}(y)$ denote the homothetic ball centered at $y$.

Theorem 1.1. Let $u$ be a non-negative, local, weak solution to (1.1)-(1.2), satisfying

$$
\left|[u(\cdot, t)>M] \cap B_{\rho}(y)\right|>\alpha\left|B_{\rho}\right|
$$

for all times

$$
s-\epsilon M^{2-p} \rho^{p} \leq t \leq s
$$

for some $M>0$, and some $\alpha$ and $\epsilon$ in $(0,1)$, and assume that

$$
B_{16 \rho} \times\left[s-\epsilon M^{2-p} \rho^{p}, s\right] \subset E_{T} .
$$

There exist $\sigma \in(0,1)$ and $\varepsilon \in\left(0, \frac{1}{2} \epsilon\right]$, which can be determined a priori, quantitatively only in terms of the data, and the numbers $\alpha$ and $\epsilon$, and independent of $M$, such that

$$
u(x, t) \geq \sigma M \quad \text { for all } x \in B_{2 \rho}(y)
$$

for all times

$$
s-\varepsilon M^{2-p} \rho^{p}<t \leq s .
$$

Thus measure-theoretical information on the measure of the "positivity set" in $B_{\rho}(y)$ for all times in (1.4) implies that such a positivity set actually "expands" to $B_{2 \rho}(y)$ for comparable times. This fact is at the heart of any form of Harnack estimate, as evidenced in [3, 4]. Its proof, first given in [1] and reported in 2], Chapter IV, $\S 5$, is rather involved and not intuitive.

The goal of this paper is to give a new proof, which is more direct, being based on geometrical ideas.

\section{Preliminaries}

For positive $\rho$ and $\theta$ set

$$
Q_{\rho}^{-}(\theta)=B_{\rho} \times\left(-\theta \rho^{p}, 0\right], \quad Q_{\rho}^{+}(\theta)=B_{\rho} \times\left(0, \theta \rho^{p}\right]
$$

and for $(y, s) \in \mathbb{R}^{N} \times \mathbb{R}$,

$$
\begin{aligned}
& (y, s)+Q_{\rho}^{-}(\theta)=B_{\rho}(y) \times\left(s-\theta \rho^{p}, s\right], \\
& (y, s)+Q_{\rho}^{+}(\theta)=B_{\rho}(y) \times\left(s, s+\theta \rho^{p}\right] .
\end{aligned}
$$

There exists a constant $\gamma=\gamma($ data $)$ such that, for every cylinder $\left[(y, s)+Q_{\rho}^{-}(\theta)\right] \subset$ $E_{T}$, every $k \in \mathbb{R}$ and every piecewise smooth, non-negative function $\zeta$ vanishing on $\partial B_{\rho}(y)$

$$
\begin{aligned}
\underset{s-\theta \rho^{p}<t<s}{\operatorname{essuup}} & \int_{B_{\rho}(y)}(u-k)_{ \pm}^{2} \zeta^{p}(x, t) d x-\int_{B_{\rho}(y)}(u-k)_{ \pm}^{2} \zeta^{p}\left(x, s-\theta \rho^{p}\right) d x \\
& +C_{o} \iint_{(y, s)+Q_{\rho}^{-}(\theta)}\left|D(u-k)_{ \pm} \zeta\right|^{p} d x d \tau \\
& \leq \gamma \iint_{(y, s)+Q_{\rho}^{-}(\theta)}\left[(u-k)_{ \pm}^{p}|D \zeta|^{p}+(u-k)_{ \pm}^{2}\left|\zeta_{\tau}\right|\right] d x d \tau
\end{aligned}
$$

where $C_{o}$ is the constant appearing in the structure conditions. Similar energy estimates continue to hold for cylinders $\left[(y, s)+Q_{\rho}^{+}(\theta)\right] \subset E_{T}$. 
Remark 2.1. The constant $\gamma(p) \rightarrow \infty$ as $p \rightarrow 1$, but it is stable as $p \rightarrow 2$. Thus (2.1) holds for all $1<p \leq 2$, with the indicated behavior of the constant $\gamma(p)$.

\subsection{A DeGiorgi-type lemma.}

Lemma 2.2. There exists a number $\nu$ depending upon the data $\theta$ and $M$, such that if

then

$$
\left|[u \leq M] \cap\left[(y, s)+Q_{2 \rho}^{-}(\theta)\right]\right| \leq \nu\left|Q_{2 \rho}^{-}(\theta)\right|
$$

$$
u \geq \frac{1}{2} M \quad \text { a.e. in }\left[(y, s)+Q_{\rho}^{-}(\theta)\right]
$$

Proof. Without loss of generality, we may assume that $(y, s)=(0,0)$. For $n=$ $0,1,2, \ldots$, set

$$
\rho_{n}=\rho+\frac{\rho}{2^{n}}, \quad B_{n}=B_{\rho_{n}}, \quad Q_{n}=B_{n} \times\left(-\theta \rho_{n}^{p}, 0\right] .
$$

Apply (2.1) over $B_{n}$ and $Q_{n}$ to $\left(u-k_{n}\right)_{-}$, for the levels

$$
k_{n}=\left(\frac{1}{2}+\frac{1}{2^{n}}\right) M
$$

The cutoff function $\zeta$ is taken to be of the form $\zeta(x, t)=\zeta_{1}(x) \zeta_{2}(t)$, where

$$
\begin{array}{ll}
\zeta_{1}= \begin{cases}1 & \text { in } B_{n+1}, \\
0 & \text { in } \mathbb{R}^{N}-B_{n},\end{cases} & \left|D \zeta_{1}\right| \leq \frac{1}{\rho_{n}-\rho_{n+1}}=\frac{2^{n+1}}{\rho}, \\
\zeta_{2}= \begin{cases}0 & \text { for } t<-\theta \rho_{n}^{p}, \\
1 & \text { for } t \geq-\theta \rho_{n+1}^{p},\end{cases} & 0 \leq \zeta_{2, t} \leq \frac{1}{\theta\left(\rho_{n}^{p}-\rho_{n+1}^{p}\right)} \leq \frac{2^{p(n+1)}}{\theta \rho^{p}} .
\end{array}
$$

The energy inequalities (2.1) with these stipulations yield

$$
\begin{aligned}
\underset{-\theta \rho_{n}^{p}<t<0}{\operatorname{ess} \sup _{0}} & \int_{B_{n}}\left(u-k_{n}\right)_{-}^{2} \zeta^{2}(x, t) d x+\iint_{Q_{n}}\left|D\left(u-k_{n}\right)_{-} \zeta\right|^{p} d x d \tau \\
& \leq \gamma \frac{2^{n p}}{\rho^{p}}\left(\iint_{Q_{n}}\left(u-k_{n}\right)_{-}^{p} d x d \tau+\frac{1}{\theta} \iint_{Q_{n}}\left(u-k_{n}\right)_{-}^{2} d x d \tau\right) \\
& \leq \gamma \frac{2^{n p}}{\rho^{p}}\left(M^{p}+\frac{M^{2}}{\theta}\right)\left|\left[u<k_{n}\right] \cap Q_{n}\right| .
\end{aligned}
$$

By Proposition 3.1 of [2], Chapter I,

$$
\begin{aligned}
& \iint_{Q_{n}}\left[\left(u-k_{n}\right)_{-} \zeta\right]^{p \frac{N+2}{N}} d x d t \\
& \quad \leq \iint_{Q_{n+1}} \mid D\left[( u - k _ { n } ) _ { - } \zeta | ^ { p } d x d \tau \left(\underset{-\theta \rho_{n}^{p}<t<0}{\left.\operatorname{ess} \sup _{B_{n}(t)}\left[\left(u-k_{n}\right)_{-} \zeta\right]^{2} d x\right)^{\frac{p}{N}}}\right.\right. \\
& \quad \leq \gamma\left[\frac{2^{n p}}{\rho^{p}}\left(M^{p}+\frac{M^{2}}{\theta}\right)\right]^{\frac{N+p}{N}}\left|\left[u<k_{n}\right] \cap Q_{n}\right|^{\frac{N+p}{N}} .
\end{aligned}
$$

Estimate

$$
\iint_{Q_{n}}\left[\left(u-k_{n}\right)-\zeta\right]^{p \frac{N+2}{N}} d x d t \geq\left[2^{-(n+2)} M\right]^{p \frac{N+2}{N}}\left|\left[u<k_{n+1}\right] \cap Q_{n+1}\right|
$$

and set

$$
Y_{n}=\frac{\left|\left[u<k_{n}\right] \cap Q_{n}\right|}{\left|Q_{n}\right|} .
$$


Then

where

$$
Y_{n+1} \leq \gamma b^{n}\left(\frac{\theta}{M^{2-p}}\right)^{\frac{p}{N}}\left(1+\frac{M^{2-p}}{\theta}\right)^{\frac{N+p}{N}} Y_{n}^{1+\frac{p}{N}}
$$

$$
b=2^{\frac{p}{N}[2(N+1)+p]} \quad \text { and } \quad \gamma=\gamma(\text { data }) .
$$

By Lemma 4.1 of [2], Chapter I, $\left\{Y_{n}\right\} \rightarrow 0$ as $n \rightarrow \infty$, provided that

$$
Y_{o} \leq \gamma_{o} \frac{\frac{M^{2-p}}{\theta}}{\left(1+\frac{M^{2-p}}{\theta}\right)^{\frac{N+p}{p}}} \stackrel{\text { def }}{=} \nu, \quad \text { where } \gamma_{o}=\frac{1}{\gamma^{\frac{N}{p}}} \frac{1}{b^{\left(\frac{N}{p}\right)^{2}}} .
$$

Thus this choice of $\nu$ yields $Y_{\infty}=0$, which is equivalent to (2.2).

2.2. A variant of Lemma 2.2. Assume now that some information is available on the "initial data" relative to the cylinder $\left[(y, s)+Q_{2 \rho}^{+}(\theta)\right]$, say, for example,

$$
u(x, s) \geq M \quad \text { for a.e. } x \in B_{2 \rho}(y)
$$

for some $M>0$. Then, writing the energy inequalities (2.1) for $(u-k)_{-}$, for $k \leq M$, over the cylinder $\left[(y, s)+Q_{2 \rho}^{+}(\theta)\right]$, the integral extended over $B_{2 \rho}$, at the time level $t=s$, vanishes in view of (2.3). Moreover, by taking cutoff functions $\zeta(x, t)=\zeta_{1}(x)$ independent of $t$, also the integral involving $\zeta_{t}$ on the right-hand side of (2.1) vanishes. We may now repeat the same arguments as in the previous proof for $\left(u-k_{n}\right)_{-}$, over the cylinders $Q_{n}^{+}=B_{n} \times\left(0, \theta(2 \rho)^{p}\right]$.

This leads to similar estimates as before, with $Q_{n}$ replaced by $Q_{n}^{+}$, and with $A_{n}$ replaced by $A_{n}^{+}=\left[u<\xi_{n} M\right] \cap Q_{n}^{+}$. Proceeding as before gives

$$
Y_{n+1}^{+} \leq \gamma b^{n}\left(\frac{\theta}{M^{2-p}}\right)^{\frac{p}{N}}\left(Y_{n}^{+}\right)^{1+\frac{p}{N}}
$$

where $Y^{+}=\left|A_{n}^{+}\right| /\left|Q_{n}^{+}\right|$and $b=2^{\frac{p}{N}(2 N+2+p)}$. This in turn implies that $\left\{Y_{n}^{+}\right\} \rightarrow 0$ as $n \rightarrow \infty$, provided that

$$
Y_{o}^{+} \leq \delta \frac{M^{2-p}}{\theta}
$$

for a constant $\delta \in(0,1)$ depending only upon the data, and independent of $M, \rho$, and $\theta$. We summarise.

Lemma 2.3. Let $M$ be a positive number such that both (2.3) and (2.4) hold. Then

$$
u \geq \frac{1}{2} M \quad \text { a.e. in } B_{\rho}(y) \times\left(s, s+\theta(2 \rho)^{p}\right] .
$$

Remark 2.4. Both Lemmas 2.2 and 2.3 are based on the energy estimates (2.1) and Proposition 3.1 of [2], Chapter I, which continue to hold in a stable manner for $p \rightarrow 2$. These results are therefore valid for all $1<p \leq 2$.

\section{Transforming the Variables and the PDE}

Fix $(y, s) \in E_{T}$, and let $\rho>0$ be so that the cylindrical domain

$$
B_{16 \rho} \times\left(s-\epsilon M^{2-p} \rho^{p}, s\right) \subset E_{T} .
$$

Introduce the change of variables and the new unknown function

$$
z=\frac{x-y}{\rho}, \quad-e^{-\tau}=\frac{t-s}{\epsilon M^{2-p} \rho^{p}}, \quad v(z, \tau)=\frac{1}{M} u(x, t) e^{\frac{\tau}{2-p}} .
$$


This maps the cylinder in (3.1) into $B_{16} \times(0, \infty)$ and transforms (1.1) into

$$
v_{\tau}-\operatorname{div} \overline{\mathbf{A}}\left(z, \tau, v, D_{z} v\right)=\frac{1}{2-p} v \quad \text { weakly in } B_{16} \times(0, \infty),
$$

where $\overline{\mathbf{A}}$ is a measurable vector-valued function of its arguments, satisfying the structure condition

$$
\left\{\begin{array}{l}
\overline{\mathbf{A}}\left(z, \tau, v, D_{z} v\right) \cdot D_{z} v \geq \epsilon C_{o}\left|D_{z} v\right|^{p} \\
\left|\overline{\mathbf{A}}\left(z, \tau, v, D_{z} v\right)\right| \leq \epsilon C_{1}\left|D_{z} v\right|^{p-1}
\end{array} \quad \text { a.e. in } B_{16} \times(0, \infty)\right.
$$

where $C_{o}$ and $C_{1}$ are the constants in the structure conditions (1.2) and $\epsilon$ is the number appearing in (1.4). The assumption (1.3) of Theorem 1.1 becomes

$$
\left|\left[v(\cdot, \tau) \geq e^{\frac{\tau}{2-p}}\right] \cap B_{1}\right| \geq \alpha\left|B_{1}\right| \quad \text { for all } \tau \in(0,+\infty) .
$$

Let $\tau_{o}>0$ be chosen and set

$$
k=e^{\frac{\tau_{o}}{2-p}}, \quad \text { and } k_{j}=\frac{1}{2^{j}} k \quad \text { for } j=0,1, \ldots
$$

With this symbolism (3.5) implies that

$$
\left|[v(\cdot, \tau) \geq k] \cap B_{8}\right| \geq \alpha 8^{-N}\left|B_{8}\right| \quad \text { for all } \tau \in\left(\tau_{o},+\infty\right) .
$$

Introduce the cylinders

$$
\begin{aligned}
& Q_{\tau_{o}}=B_{8} \times\left(\tau_{o}+k^{2-p}, \tau_{o}+2 k^{2-p}\right), \\
& Q_{\tau_{o}}^{\prime}=B_{16} \times\left(\tau_{o}, \tau_{o}+2 k^{2-p}\right)
\end{aligned}
$$

and a piecewise smooth cutoff function on $Q_{\tau_{o}}^{\prime}$ of the form $\zeta(z, \tau)=\zeta_{1}(z) \zeta_{2}(\tau)$, where

$$
\begin{array}{ll}
\zeta_{1}=\left\{\begin{array}{lll}
1 & \text { in } B_{8} & \\
0 & \text { in } \mathbb{R}^{N}-B_{16} & D \zeta_{1} \mid \leq \frac{1}{8},
\end{array}\right. \\
\zeta_{2}=\left\{\begin{array}{lll}
0 & \text { for } \tau<\tau_{o} \\
1 & \text { for } \tau \geq \tau_{o}+k^{2-p} & 0 \leq \zeta_{2, \tau} \leq \frac{1}{k^{2-p}} .
\end{array}\right.
\end{array}
$$

Energy estimates, over $Q_{\tau_{o}}^{\prime}$, are derived by taking $-\left(v-k_{j}\right)-\zeta^{p}$ as a testing function in the weak formulation of (3.3). Discarding the non-positive contribution of the right-hand side, standard calculations give

$$
\int_{Q_{\tau_{o}}^{\prime}}\left|D\left(v-k_{j}\right)_{-} \zeta\right|^{p} d z d \tau \leq \gamma \int_{Q_{\tau_{o}}^{\prime}}\left[\left(v-k_{j}\right)_{-}^{p}|D \zeta|^{p}+\left(v-k_{j}\right)_{-}^{2}\left|\zeta_{\tau}\right|\right] d z d \tau
$$

where the constant $\gamma$ depends only upon $N, p, C_{o}, C_{1}$ and the parameter $\epsilon$ appearing in the assumption (1.4) of Theorem 1.1 and the transformed structure conditions (3.4). From this

$$
\int_{Q_{\tau_{o}}}\left|D\left(v-k_{j}\right)_{-}\right|^{p} d z d \tau \leq 2 \gamma k_{j}^{p}\left|Q_{\tau_{o}}\right|
$$


3.1. Estimating the measure of the set $\left[v<k_{j}\right]$ within $Q_{\tau_{o}}$. Set

$$
A_{j}(\tau)=\left[v(\cdot, \tau)<k_{j}\right] \cap B_{8}, \quad A_{j}=\left[v<k_{j}\right] \cap Q_{\tau_{o}} .
$$

By the measure-theoretical Lemma 2.2 of [2], Chapter I, and (3.6),

$$
\begin{aligned}
\left(k_{j}-k_{j+1}\right)\left|A_{j+1}(\tau)\right| & \leq \frac{\gamma(N)}{\left|B_{8}-A_{j}(\tau)\right|} \int_{\left[k_{j+1}<v(\cdot, \tau)<k_{j}\right]}|D v| d z \\
& \leq \gamma(N, \alpha) \int_{\left[k_{j+1}<v(\cdot, \tau)<k_{j}\right]}|D v| d z
\end{aligned}
$$

for all $\tau \geq \tau_{o}$. Integrate this in $d \tau$ over $\left(\tau_{o}+k^{2-p}, \tau_{o}+2 k^{2-p}\right)$, majorize the resulting integral on the right-hand side by the Hölder inequality, and use (3.7) to get

$$
\begin{aligned}
\frac{k_{j}}{2}\left|A_{j+1}\right| & \leq \gamma(\text { data, } \alpha) \int_{\left[k_{j+1}<v<k_{j}\right]}|D v| d z d \tau \\
& \leq \gamma(\text { data, } \alpha)\left(\int_{\left[k_{j+1}<v<k_{j}\right]}|D v|^{p} d z d \tau\right)^{\frac{1}{p}}\left|A_{j}-A_{j+1}\right|^{\frac{p-1}{p}} \\
& \leq \gamma(\text { data, } \alpha, \epsilon)\left(\int_{Q_{\tau_{o}}}\left|D\left(v-k_{j}\right)_{-}\right|^{p} d z d \tau\right)^{\frac{1}{p}}\left|A_{j}-A_{j+1}\right|^{\frac{p-1}{p}} \\
& \leq \gamma(\text { data, } \alpha, \epsilon) k_{j}\left|Q_{\tau_{o}}\right|^{\frac{1}{p}}\left|A_{j}-A_{j+1}\right|^{\frac{p-1}{p}} .
\end{aligned}
$$

Taking the $p /(p-1)$-power yields the recursive inequalities

$$
\left|A_{j+1}\right|^{\frac{p}{p-1}} \leq \gamma(\text { data, } \alpha, \epsilon)|Q|^{\frac{1}{p-1}}\left|A_{j}-A_{j+1}\right| .
$$

Add these inequalities for $j=0,1, \ldots, j_{*}-1$, where $j_{*}$ is an integer to be chosen, and majorize the sum on the right-hand side by the corresponding telescopic series. This gives

Equivalently

$$
\left(j_{*}-1\right)\left|A_{j_{*}}\right|^{\frac{p}{p-1}} \leq \gamma(\text { data }, \alpha, \epsilon)|Q|^{\frac{p}{p-1}} .
$$

$$
\left|\left[v<k_{j_{*}}\right] \cap Q_{\tau_{o}}\right| \leq \nu\left|Q_{\tau_{o}}\right|, \quad \text { where } \quad \nu=\left(\frac{\gamma(\text { data }, \alpha, \epsilon)}{j_{*}}\right)^{\frac{p-1}{p}} .
$$

Remark 3.1. This estimation technically requires $p>1$. The restriction however is not only technical in view of the geometrical significance of (1.1) for $p=1$ even in the elliptic case (5]).

3.2. Segmenting $Q_{\tau_{o}}$. Assume momentarily that $j_{*}$ and hence $\nu$ has been determined. By possibly increasing $j_{*}$ to be not necessarily integer, we may assume, without loss of generality, that $\left(2^{j_{*}}\right)^{2-p}$ is an integer. Then subdivide $Q_{\tau_{o}}$ into $\left(2^{j_{*}}\right)^{2-p}$ cylinders, each of length $k_{j_{*}}^{2-p}$, by setting

$$
\begin{aligned}
& Q_{n}=B_{8} \times\left(\tau_{o}+k^{2-p}+n k_{j_{*}}^{2-p}, \tau_{o}+k^{2-p}+(n+1) k_{j_{*}}^{2-p}\right) \\
& \text { for } n=0,1, \ldots,\left(2^{j_{*}}\right)^{2-p}-1 .
\end{aligned}
$$

For at least one of these, say $Q_{n}$, we must have

$$
\left|\left[v<k_{j_{*}}\right] \cap Q_{n}\right| \leq \nu\left|Q_{n}\right| .
$$

Apply Lemma 2.2 to $v$ over $Q_{n}$ with $M=k_{j_{*}}$ and $\theta=k_{j_{*}}^{2-p}$. This gives

$$
v\left(z, \tau_{o}+k^{2-p}+(n+1) k_{j_{*}}^{2-p}\right) \geq \frac{1}{2} k_{j_{*}} \quad \text { a.e. in } B_{4}
$$


provided that

$$
\frac{\left|\left[v<k_{j_{*}}\right] \cap Q_{n}\right|}{\left|Q_{n}\right|} \leq 2^{-\frac{N+p}{p}} \bar{\gamma}_{o}(\text { data }, \epsilon)=\nu .
$$

Now choose $j_{*}$, and hence $\nu$, from this and (3.8). Summarising, for such a choice of $j_{*}$, and hence $\nu$, there exists a time level $\tau_{1}$ in the range

$$
\tau_{o}+k^{2-p}<\tau_{1}<\tau_{o}+2 k^{2-p}
$$

such that

$$
v\left(z, \tau_{1}\right) \geq \sigma_{o} e^{\frac{\tau_{o}}{2-p}}, \quad \text { where } \quad \sigma_{o}=2^{-\left(j_{*}+1\right)} .
$$

Remark 3.2. Notice that $j_{*}$ and hence $\nu$ are determined only in terms of the data and are independent of the parameter $\tau_{o}$, which is still to be chosen.

3.3. Returning to the original coordinates. In terms of the original coordinates and the original function $u(x, t)$, this implies that

$$
u\left(\cdot, t_{1}\right) \geq \sigma_{o} M e^{-\frac{\tau_{1}-\tau_{o}}{2-p}} \stackrel{\text { def }}{=} M_{o} \quad \text { in } B_{4 \rho}(y),
$$

where the time $t_{1}$ corresponding to $\tau_{1}$ is computed from (3.2) and (3.9). Now apply Lemma 2.3 with $M$ replaced by $M_{o}$ over the cylinder

$$
\left(t_{1}, y\right)+Q_{4 \rho}^{+}=B_{4 \rho}(y) \times\left(t_{1}, t_{1}+\theta(4 \rho)^{p}\right] .
$$

By choosing

$$
\theta=\delta M_{o}^{2-p}, \quad \text { where } \quad \delta=\delta(\text { data }),
$$

the assumption (2.4) is satisfied, and Lemma 2.3 yields

$$
\begin{aligned}
u(\cdot, t) & \geq \frac{1}{2} M_{o}=\frac{1}{2} \sigma_{o} M e^{-\frac{\tau_{1}-\tau_{o}}{2-p}} \quad \text { in } \quad B_{2 \rho}(y) \\
& \geq \frac{1}{2} \sigma_{o} e^{-\frac{2}{2-p} e^{\tau_{o}}} M
\end{aligned}
$$

for all times

$$
t_{1} \leq t \leq t_{1}+\delta M_{o}^{2-p}(2 \rho)^{p} .
$$

If the right-hand side equals $s$, then (3.10) holds for all times in

$$
\left(s-\varepsilon M^{2-p}(2 \rho)^{p}, s\right], \quad \text { where } \quad \varepsilon=\delta \sigma_{o}^{2-p} e^{-e^{\tau_{o}}} .
$$

Thus the conclusion of Theorem 1.1 holds, provided that the upper time level in (3.11) equals $s$. The transformed $\tau_{o}$ level is still undetermined, and it will be so chosen as to verify such a requirement. Precisely, taking into account (3.2),

$$
\epsilon M^{2-p} \rho^{p} e^{-\tau_{1}}=-\left(t_{1}-s\right)=\delta \sigma_{o} M^{2-p}(2 \rho)^{p} e^{-\left(\tau_{1}-\tau_{o}\right)} \quad \Longrightarrow \quad e^{\tau_{o}}=\frac{\epsilon}{2^{p} \delta \sigma_{o}} .
$$

This determines quantitatively $\tau_{o}=\tau_{o}$ (data). The proof of Theorem 1.1 is now completed by inserting such a $\tau_{o}$ on the right-hand side of (3.10) and (3.12). 


\section{Stability FOR $p \approx 2$}

The change of variables (3.2) and the subsequent arguments yield constants that deteriorate as $p \rightarrow 2$. The conclusion of Theorem 1.1 however continues to hold with constants that are "stable" as $p \rightarrow 2$. We indicate here the modifications of the arguments needed to achieve such a constant stability.

For $p \approx 2$, instead of (3.2) introduce the change of variables

$$
z=\frac{x-y}{\rho}, \quad \tau=\frac{t-s}{\epsilon M^{2-p} \rho^{p}}, \quad v(z, \tau)=\frac{1}{M} u(x, t) .
$$

This maps the cylinder in (3.1) into $B_{16} \times(-1,0)$ and transforms (1.1) into

$$
v_{\tau}-\operatorname{div} \overline{\mathbf{A}}\left(z, \tau, v, D_{z} v\right)=0 \quad \text { weakly in } B_{16} \times(-1,0),
$$

where $\overline{\mathbf{A}}$ satisfies (3.4) over the indicated cylinder. The arguments of $\$ 3.1$ are intended to lead to (3.8) so that $j_{*}$ and hence $\nu$ could be chosen so as to satisfy the requirement (2.2) of Lemma 2.2, quantified by (2.1). Now the arguments of 93.1 can be repeated here with the proper modifications, where $\left\{Q_{n}\right\}$ now forms a nested collection of cubes in $B_{8} \times(-1,0)$ with the same vertex at $(0,0)$, with $k$ replaced by 1 and hence $k_{j}=2^{-j}$. This leads to (3.8) with $Q_{\tau_{o}}$ replaced by $B_{4} \times(-1,0)$. By Lemma 2.2 and (2.1), this would imply that $v \geq \frac{1}{2} 2^{-j_{*}}$ in $B_{2} \times\left(-\frac{1}{2}, 0\right)$ provided that

$$
\left[v<k_{j_{*}}\right] \cap B_{4} \times(-1,0) \mid \leq \gamma_{o} \frac{2^{-j_{*}(2-p)}}{\left(1+2^{-j_{*}(2-p)}\right)^{\frac{N+p}{p}}}
$$

for a constant $\gamma_{o}=\gamma_{o}$ (data). From (3.8) with indicated modifications, this is possible if one can choose $j_{*} \in \mathbb{N}$ so that

$$
\nu=\left(\frac{\gamma(\text { data }, \alpha, \epsilon)}{j_{*}}\right)^{\frac{p-1}{p}} \leq \gamma_{o} \frac{2^{-j_{*}(2-p)}}{\left(1+2^{-j_{*}(2-p)}\right)^{\frac{N+p}{p}}} .
$$

This is possible if $p$ is sufficiently close to 2 by the following procedure. First choose $\left(2-p_{o}\right)=1 / j_{*}$, and then $j_{*}$ so large as to satisfy the indicated requirement for all $p_{o} \leq p \leq 2$.

\section{Equations of POROUS-MEdium type}

The techniques apply, by minor variants, to non-negative solutions of quasilinear, singular, parabolic equations of the porous-medium type

$$
\begin{array}{ll}
u \in C_{\mathrm{loc}}\left(0, T ; L_{\mathrm{loc}}^{m+1}(E)\right),|u|^{m} \in L_{\mathrm{loc}}^{2}\left(0, T ; W_{\mathrm{loc}}^{1,2}(E)\right), & 0<m<1 . \\
u_{t}-\operatorname{div} \mathbf{A}(x, t, u, D u)=0 & \text { weakly in } E_{T},
\end{array}
$$

The functions $\mathbf{A}: E_{T} \times \mathbb{R}^{N+1} \rightarrow \mathbb{R}^{N}$ are only assumed to be measurable and subject to the structure conditions

$$
\left\{\begin{array}{l}
\mathbf{A}(x, t, u, D u) \cdot D u \geq\left.\left. C_{o}|u|^{1-m}|D| u\right|^{m}\right|^{2} \\
|\mathbf{A}(x, t, u, D u)| \leq\left. C_{1}|D| u\right|^{m} \mid
\end{array} \quad \text { a.e. in } E_{T},\right.
$$

where $C_{o}$ and $C_{1}$ are given positive constants. The homogeneous prototype is

$$
u_{t}-\Delta|u|^{m-1} u=0, \quad 0<m<1, \quad \text { weakly in } E_{T} .
$$


Theorem 5.1. Let u be a non-negative, local, weak solution to (5.1)-(5.2) satisfying

$$
\left|[u(\cdot, t)>M] \cap B_{\rho}(y)\right|>\alpha\left|B_{\rho}\right|
$$

for all times

$$
s-\epsilon M^{1-m} \rho^{2} \leq t \leq s
$$

for some $M>0$ and some $\alpha$ and $\epsilon$ in $(0,1)$, and assume that

$$
B_{16 \rho} \times\left[s-\epsilon M^{1-m} \rho^{2}, s\right] \subset E_{T} .
$$

There exist $\sigma \in(0,1)$ and $\varepsilon \in\left(0, \frac{1}{2} \epsilon\right]$, which can be determined a priori, quantitatively only in terms of the data, and the numbers $\alpha$ and $\epsilon$, and independent of $M$, such that

$$
u(x, t) \geq \sigma M \quad \text { for all } x \in B_{2 \rho}(y)
$$

for all times

$$
s-\varepsilon M^{1-m} \rho^{2}<t \leq s .
$$

Thus measure-theoretical information on the measure of the "positivity set" in $B_{\rho}(y)$ for all times in (5.4) implies that such a positivity set actually "expands" to $B_{2 \rho}(y)$ for comparable times. Applications of such a "spread of positivity" to Harnack estimates for non-negative solutions of porous-medium equations of the type (5.1)-(5.2) are in [3, 4].

\section{REFERENCES}

1. Y.Z. Chen; E. DiBenedetto, Hölder estimates of solutions of singular parabolic equations with measurable coefficients. Arch. Rational Mech. Anal., 118(3) (1992), 257-271. MR.1158938 (93a:35092)

2. E. DiBenedetto, Degenerate Parabolic Equations. Universitext, Springer-Verlag, New York, 1993. MR1230384 (94h:35130)

3. E. DiBenedetto; U. Gianazza; V. Vespri, Harnack Estimates for Quasi-Linear Degenerate Parabolic Differential Equations. Acta Math., 200 (2008), 181-209. MR2413134 (2009g:35130)

4. E. DiBenedetto; U. Gianazza; V. Vespri, Forward, Backward and Elliptic Harnack Inequalities for Non-Negative Solutions to Certain Singular Parabolic Partial Differential Equations. Ann. Scuola Norm. Super. Pisa Cl. Sci. (5) (in press).

5. M. Miranda, Sul minimo dell'integrale del gradiente di una funzione. Ann. Scuola Norm. Sup. Pisa, 3(19) (1965), 626-665. MR0188839(32:6271)

Department of Mathematics, Vanderbilt University, 1326 Stevenson Center, NashVILle, Tennessee 37240

E-mail address: em.diben@vanderbilt.edu

Dipartimento di Matematica "F. Casorati", Università di Pavia, via Ferrata 1, 27100 PAVIA, ITALY

E-mail address: gianazza@imati.cnr.it

Dipartimento di Matematica "U. Dini", Università di Firenze, viale Morgagni 67/A, 50134 FiRENZE, ItALY

E-mail address: vespri@math.unifi.it 